\title{
DEFICIENCIAS EPISTEMOLÓGICAS EN LA \\ ENSEÑANZA DE LAS REACCIONES ÁCIDO-BASE \\ Y DIFICULTADES DE APRENDIZAJE
}

\author{
Carlos Furió \\ Departamento de Didáctica de las Ciencias Experimentales y \\ Sociales. \\ Universidad de Valencia (España). \\ $M^{a}$ Luisa Calatayud \\ Instituto Enseñanza Secundaria "Sorolla" de Valencia (España). \\ Sergio Bárcenas \\ Escuela Preparatoria de la Universidad de Zacatecas
}

(México).

\begin{abstract}
The aim of this work is to find out in what way distorted visions of chemistry (linear accumulative and non-problematic) in the teaching can explain the lack a meaningful learning in the study of reactions between acids and bases. The students have difficulties in the neutralization and the hydrolysis due, between other reasons, to epistemological and methodological deficiencies by teaching process not taking into account the results of research in science education.
\end{abstract}

\begin{abstract}
RESUMEN
El objetivo de este trabajo consiste en mostrar cómo la existencia de visiones distorsionadas de las Química, como la acumulación lineal y la problemática, puede explicar la falta de aprendizaje significativo en el estudio de las reacciones entre ácidos y bases. En efecto, se comprueba que los estudiantes tienen dificultades en los procesos de neutralización e hidrólisis, debido, entre otras razones, a deficiencias epistemológicas y metodológicas en una enseñanza que no tiene en cuenta los resultados de la investigación didáctica.
\end{abstract}

\section{INTRODUCCIÓN}

Uno de los temas importantes de Química del bachillerato de Ciencias es el de las reacciones ácido-base y, sin embargo, las investigaciones realizadas en Didáctica de las Ciencias sobre las dificultades en el aprendizaje de este tema representan un porcentaje insignificante según revisiones bibliográficas recientes (Pfundt y Duit, 1994). Trabajos 
como los de Cros (1986 y 1988), Zoller (1990), Schmidt (1991), Ross (1991), Nakhleh (1994) y Vidyapati y Seetharamappa (1995) muestran el pobre aprendizaje significativo logrado en este tema. La literatura citada no comenta la existencia de concepciones alternativas que puedan obstaculizar el aprendizaje y, por tanto, es lógico suponer una mayor responsabilidad de la enseñanza en el fracaso escolar (Gabel, 1998).

El presente trabajo tiene como objetivo averiguar en qué medida una enseñanza basada en visiones distorsionadas de la química y de su aprendizaje puede explicar la falta de aprendizaje significativo de las reacciones entre ácidos y bases. La hipótesis emitida supone aceptar que los estudiantes de Química van a tener serias dificultades en el aprendizaje de las reacciones de neutralización y de hidrólisis debido, fundamentalmente, a las carencias e insuficiencias epistemológicas y metodológicas de la enseñanza de estos conceptos al no tener en cuenta los resultados de la investigación en la Didáctica de las Ciencias.

\section{VISIONES DISTORSIONADAS DE LA QUÍMICA EN LA ENSEÑANZA DE LAS REACCIONES ÁCIDO-BASE E IMPLICACIONES DIDÁCTICAS}

Las investigaciones recientes en la formación del profesorado han puesto de relieve la existencia de un pensamiento docente espontáneo sobre la Ciencia y la actividad científica de 'sentido común' que conviene conocer y cuestionar porque puede significar un serio obstáculo a la renovación didáctica del propio profesorado (Furió, 1994). No se ha estudiado todavía cómo la transmisión tácita de errores epistemológicos concretos en la enseñanza de estos procesos puede contribuir a agudizar las dificultades de los estudiantes en una temática determinada. A continuación se han seleccionado por la importancia de sus implicaciones didácticas, algunas de estas deficiencias epistemológicas del profesorado a la luz de los resultados actuales de la investigación.

\subsection{Visión acumulativa lineal de las teorías científicas y sus implicaciones en el aprendizaje}

De acuerdo con Gil (1993) la visión acumulativa lineal de la Ciencia consiste principalmente en la creencia de que el conocimiento científico se va descubriendo e incorporando linealmente a los ya existentes. Esta creencia se deriva de una visión empirista de la ciencia donde se supone que cada teoría descubierta es la misma que la anterior pero más general. Así en la enseñanza de las reacciones ácido-base es frecuente presentar, por ejemplo, la teoría de la transferencia protónica de Brönsted y Lowry (1923) como la de la disociación iónica de Arrhenius (1884) ampliada. Sin embargo, es sabido que cuando se presentan muchos problemas a una teoría y es sustituida por otra nueva más general, cambian de significado los conceptos nucleares de la vieja teoría (Kuhn 1973). Así pues, si no queremos caer en una visión acumulativa lineal tendremos que analizar con detalle los cambios teóricos habidos en este dominio hasta llegar a la teoría de Brönsted para ver cómo han cambiado los conceptos introducidos. La historia de la evolución de las distintas teorías ácido-base es conocida superficialmente por el profesorado. En esta evolución se reconoce fácilmente la existencia de, al menos, tres perfiles conceptuales (Mortimer, 1995) para interpretar las reacciones ácido-base:

i) Perfil macroscópico o funcional de las reacciones de neutralización 
Parte de la acumulación precientífica de conocimientos fenomenológicos sobre los ácidos y las bases y su neutralización respectiva. Este perfil se puede dar por concluido cuando se define en 1728 compuesto químico por Geoffroy, pues en su tabla de afinidades de sustancias figura, entre otras, las correspondientes a la "familia de los ácidos" (Klein, 1994). Se ha de tener en cuenta que durante este siglo es cuando queda bastante claramente definido el concepto operacional o funcional de sustancia (pura) como cuerpo que tiene un conjunto de propiedades específicas e invariables que permiten su caracterización (Solsona e Izquierdo 1997, Furió et al 1999). En el caso de los ácidos y de las bases se caracteriza a estas 'familias' de sustancias a partir de sus propiedades fenomenológicas características (ataque a los metales no nobles, reacción ante indicadores, caracteres organolépticos,...). La neutralización de los ácidos por las bases o viceversa se entiende como desaparición de sus propiedades respectivas al formarse las sales. Este primer perfil macroscópico de la neutralización de ácidos y bases constituirá el referente fenomenológico que deberá explicar la teoría atómica.

\section{ii) Perfil microscópico correspondiente a la teoría de Arrhenius}

Habrá que esperar a la construcción del primer cuerpo teórico de los conocimientos químicos (teoría atómica) para explicar mediante la redistribución de átomos la reacción de neutralización (ácido + base $\leftrightarrow$ sal + agua). No obstante, hasta que no se establecen relaciones entre la teoría atómica y la electricidad a fines del XIX no se tiene un primer marco teórico que permita explicar la naturaleza eléctrica de las sustancias inorgánicas con la introducción del concepto de ion. Con ello se puede predecir la existencia de iones comunes en los ácidos y en las bases. La neutralización se explica como la combinación de los respectivos iones comunes de los ácidos y las bases para formar agua: $\mathrm{H}^{+}+\mathrm{OH}^{-} \leftrightarrow$ $\mathrm{H}_{2} \mathrm{O}$ Por supuesto que junto a la idea de los iones es necesario construir la de la disociación iónica de las sustancias inorgánicas al disolverse en agua. Este proceso de disociación es condición necesaria pero no suficiente para poder llegar al de neutralización. Algunos de los problemas que se presentan en esta teoría se resuelven gracias a la participación del agua en la disociación. Por ejemplo: ¿cómo justificar que el gas $\mathrm{NH}_{3}$ al disolverse en agua tenga reacción básica? Se explica argumentando que el $\mathrm{NH}_{3}$ al disolverse en agua se disocia en iones $\mathrm{NH}_{4}{ }^{+}$y $\mathrm{OH}^{-}$De la misma forma se razona que la disolución de gas $\mathrm{HCl}$ en agua sea ácida, ya que el $\mathrm{HCl}$ se disocia en $\mathrm{CL}^{-}+\mathrm{H}^{+}$. También se explica el anfoterismo del $\mathrm{Al}(\mathrm{OH})_{3}, \mathrm{Zn}(\mathrm{OH})_{2}$, etc. gracias a la posibilidad de disociar protones o iones hidroxilo que se supone hay en su composición (fórmula). Otros problemas no se resuelven tan fácilmente y hay que idear hipótesis auxiliares como es el caso de la reacción ácida o básica que presentan las disoluciones acuosas de sales "neutras" como $\mathrm{Na}_{2} \mathrm{CO}_{3}, \mathrm{NH}_{4} \mathrm{Cl}, \mathrm{Na}_{2} \mathrm{~S}, \mathrm{KCN}$, etc. que no tienen iones $\mathrm{H}^{+}$o iones $\mathrm{OH}^{-}$en su fórmula. Debido a ello es necesario introducir un nuevo concepto, el de la hidrólisis de sales como nueva reacción entre estas sustancias y el agua. Este nuevo proceso se relaciona con la neutralización al considerarla reversible. Es decir, la hidrólisis sería el proceso opuesto a la neutralización en el equilibrio químico ácido + base↔sal + agua. Otros problemas no tienen solución en este marco teórico debido a que escapan a su campo de validez (reacciones gaseosas, en medios líquidos no acuosos, reacciones entre óxidos a elevadas temperaturas, etc.).

\section{iii) Perfil microscópico de las reacciones ácido-base según la teoría de Brönsted}

En esta teoría cambian los conceptos de ácido, base y de proceso entre ácidos y bases. La reacción ácido-base se interpreta como una transferencia protónica entre dos pares ácido- base conjugados y que se simboliza $A_{1}+B_{2} \leftrightarrow B_{1}+A_{2}$ En este nuevo marco 
teórico la neutralización se reinterpreta como: $\mathrm{H}_{3} \mathrm{O}^{+}\left(\mathrm{A}_{1}\right)+\mathrm{OH}^{-}\left(\mathrm{B}_{2}\right) \leftrightarrow \mathrm{H}_{2} \mathrm{O}\left(\mathrm{A}_{1}\right)+\mathrm{H}_{2} \mathrm{O}\left(\mathrm{A}_{2}\right)$. Por tanto, cualquier proceso de neutralización de ácidos y bases en disolución acuosa (según Arrhenius) seguirá siendo una reacción entre el ácido $\mathrm{H}_{3} \mathrm{O}$ y la base de Brönsted $\mathrm{OH}^{-}$. Pero ya no se podrá identificar cualquier reacción ácido-base de Brönsted con la neutralización. Por ejemplo, la disolución de $\mathrm{NH}_{3} \circ \mathrm{HCl}$ en agua serán reacciones ácidobase de Brönsted y no pueden catalogarse lógicamente como reacciones de neutralización. El problema de la hidrólisis queda resuelto como un proceso ácido-base de transferencia protónica donde el agua interviene como especie reactiva (bien como ácido o como base).

Veamos cuáles son las prácticas habituales en la enseñanza de estas reacciones ácido-base en la secundaria y qué consecuencias puede tener en el aprendizaje la transmisión de una visión acumulativa lineal de estas teorías. En la enseñanza habitual se suelen presentar las explicaciones sobre estos procesos de dos maneras. Una de ellas consiste en comenzar el tema introduciendo la teoría de Arrhenius muy brevemente y pasar a detenerse en la de Brönsted que es la que tiene mayor validez general. Otra secuenciación muy común es pasar directamente a explicar las reacciones ácido-base a partir de la teoría de Brönsted olvidando también presentar el perfil macroscópico de estos fenómenos que luego se van a tener que explicar (Johnstone, 1993). Al hacerlo así pueden incurrir en las siguientes deficiencias epistemológicas:

a) No se enfatiza suficientemente el perfil macroscópico de lo que se define funcionalmente como ácido, como base y, en particular, como neutralización de ácidos y bases. Sin este referente empírico difícil será que los estudiantes se imaginen qué es lo que se intenta explicar. Lo mismo suele suceder cuando se explica la hidrólisis. No se presenta la fenomenología de la hidrólisis como proceso en el cual al disolver una sal neutra en agua se producen anomalías: la solución resulta ácida o básica. Estas insuficiencias docentes en las que se omiten las definiciones funcionales de los conceptos correspondientes al perfil macroscópico serán la principal causa de que los estudiantes tengan dificultades para, por ejemplo, clasificar disoluciones acuosas de sustancias como ácidos o bases. Y en este sentido no dispondrán de criterios suficientemente claros para saber cuando se ha producido una neutralización o la hidrólisis de una sal.

b) Otro tipo de dificultades epistemológicas que posiblemente originan estas enseñanzas consiste en superponer el perfil macroscópico de estos fenómenos con las explicaciones de tipo microscópico (la de Arrhenius y/o la de Brönsted). Es decir, la descripción macroscópica de los ácidos y las bases se confunde con la interpretación conceptual de Arrhenius o de Brönsted. Se funden todos los perfiles, descritos antes, en un solo marco teórico que, en general, es el de Brönsted. Se comprende así que se identifique el concepto de neutralización de ácidos y bases en el marco de la teoría de Arrhenius con el concepto más general de reacción ácido-base como transferencia protónica en Brönsted. Es por tanto bastante plausible que esta misma confusión conceptual se dé en los estudiantes y, en particular, cuando se introducen demasiado rápidamente los conceptos de la teoría de Arrhenius y se pasa a la de Brönsted.

\subsection{Visión aproblemática de las reacciones ácido-base y dificultades de aprendizaje relacionadas}

Otra visión distorsionada de la Ciencia que se transmite implícitamente en la enseñanza de la Química y que puede derivarse de la visión acumulativa lineal indicada es la visión aproblemática o acrítica de las construcciones científicas. Esta visión se 
caracteriza por la introducción arbitraria y aproblemática de los conceptos y teorías químicas. El profesorado no hace ver que los conceptos son hipótesis, introducidos en una teoría, que responden por lo general a la necesidad epistemológica de resolver algún problema. Tampoco se exponen las crisis, los problemas, etc. que tiene una teoría (por ejemplo la de Arrhenius) y no se hace ver la necesidad de sustituirla por otra más útil (como, por ejemplo, la de Brönsted) que resolverá la mayor parte de los problemas de la vieja teoría. Es más, algunas veces estas imágenes acríticas de la ciencia llegan incluso a proponer que no hay que enseñar la vieja teoría porque supone un obstáculo en el aprendizaje de la nueva teoría (Cros 1988). De lo dicho se puede derivar una tercera deficiencia epistemológica:

c) Es frecuente en la enseñanza de la Química hacer una introducción arbitraria de los conceptos y teorías químicas. Es de esperar que no se presenten aquellos problemas que hicieron ver, por ejemplo, la necesidad de sustituir la teoría electrolítica de Arrhenius por la de la transferencia protónica de Brönsted. Tampoco se presentarán aquellos otros problemas que obligaron a introducir en la teoría de Arrhenius el concepto de hidrólisis para explicar la reacción ácida o básica de las sales neutras. Así pues, en el supuesto de que no se enseñe la teoría de Arrhenius por considerarla obsoleta se puede inducir inconscientemente algunos errores epistemológicos en los estudiantes. Por ejemplo, los procesos de neutralización e hidrólisis se diferencian claramente cuando se explican por primera vez en la teoría de Arrhenius. En cambio esta diferenciación desaparece en la teoría de Brönsted ya que ambas son consideradas reacciones ácido-base en este nuevo marco. Por tanto, es de esperar que si no se enfatiza la presentación del perfil macroscópico, ni se enseña la teoría de Arrhenius, los estudiantes acabaran considerando que neutralización e hidrólisis son procesos idénticos.

Por otro lado, es habitual en la enseñanza de los procesos químicos introducir rápidamente el uso simbólico de los mismos sin aproximaciones a las ideas que subyacen en los conceptos, en las fórmulas yen los esquemas de reacción. Se favorece así el aprendizaje memorístico de un simbolismo enseñado que el estudiante adapta a su pensamiento. Por ejemplo, ante situaciones en que los alumnos tienen que predecir si una sustancia (representada por una fórmula) al disolverla en agua es ácida o básica, utilizarán como criterio si hay átomos de $\mathrm{H}$ o grupos $\mathrm{OH}$, respectivamente en su composición, sin tener en cuenta si estos grupos atómicos son ionizables o no. $\mathrm{O}$, en el caso de que tengan que clasificar entre varios procesos cuáles son de neutralización, activarán el esquema ácido + base $\leftrightarrow$ sal + agua, donde el simbolismo empleado para la reversibilidad es convertido en igualdad. En consecuencia, no será difícil encontrar de nuevo que una neutralización es lo mismo que una hidrólisis ya que lo que está escrito a la izquierda del signo igual es lo mismo que lo que hay a la derecha.

\section{DISEÑOS Y RESULTADOS EXPERIMENTALES RELATIVOS A LAS DEFICIENCIAS EPISTEMOLÓGICAS ENCONTRADAS}

\subsection{Características generales del diseño}

Se ha elaborado un diseño experimental múltiple y convergente, empleando metodologías cualitativas y cuantitativas de forma complementaria para ver si desde perspectivas diferentes, los resultados obtenidos validan la hipótesis. A tal efecto se han elaborado dos instrumentos. El primero de ellos consiste en un protocolo de análisis del contenido de libros de texto con 8 ítems para ver si se presentan visiones deformadas 
sobre la actividad científica en el tema ácido-base. Este protocolo se ha aplicado a 40 libros de texto de Química de Bachillerato y de Química General universitaria. También se ha hecho uso de una entrevista estructurada sobre las metodologías de enseñanza de este tema a 20 profesores de Química de Bachillerato con 10 o más años de experiencia docente. En el caso de las entrevistas se han realizado sondeos previos con los ítems para analizar la dificultad de comprensión del enunciado de las preguntas.

\subsection{Resultados encontrados y análisis de los mismos}

A continuación se presentan y analizan los resultados que se han obtenido en la enseñanza de las reacciones ácido-base al poner a prueba las tres consecuencias anteriormente expuestas.

\subsubsection{La enseñanza no pone énfasis en la presentación macroscópica de lo que es un ácido, una base o una hidrólisis}

Para contrastar esta consecuencia se proponen los cuatro primeros ítems del protocolo de análisis de los libros de textos, cuyo objetivo es saber si los libros de texto tratan el perfil macroscópico de lo que se entiende por ácido, por base y por reacción de hidrólisis. Los resultados obtenidos se presentan en la tabla 1 (Anexo 1).

A pesar de que más de las $3 / 4$ partes de los libros de texto analizados presentan cuando menos 3 características de los ácidos y de las bases (ítem 1), sin embargo apenas se aprovechan estas propiedades para clasificar funcionalmente las sustancias (o sus disoluciones) en ácidas, básicas o neutras pues sólo lo hace el $18 \%$ de los textos (ítem 2). En particular, se destaca que un $60 \%$ de los textos omite la conductividad eléctrica como propiedad que puede orientar la búsqueda de explicaciones basadas en la existencia de iones. En los resultados del ítem 3 llama la atención la ausencia de ejemplos concretos de disoluciones de gases, líquidos o sólidos $(87 \%)$ conocidos familiarmente que pueden conectar la vida cotidiana y el pensamiento del estudiante con la ciencia que se estudia.

Para contrastar la consecuencia (a) propuesta respecto a que no se hace una presentación macroscópica del proceso de hidrólisis se propone el ítem 4 del protocolo de análisis de texto y la pregunta 1 de la entrevista. El objetivo de ambos es saber si en los libros de texto y silos profesores hacen un tratamiento de la hidrólisis desde el perfil macroscópico.

Un $75 \%$ de los libros de texto no presentan la hidrólisis de las sales neutras como proceso en el que la disolución obtenida tiene reacción ácida o básica. Análogos resultados se obtienen en el ítem 1 de la entrevista a los profesores.

1) ¿Cómo justificas la introducción del proceso de hidrólisis cuando lo enseñas en este tema? a) ¿Bajo qué marco teórico introduces el proceso de hidrólisis?

Catorce de 20 profesores entrevistados no presentan inicialmente la fenomenología de la hidrólisis antes de intentar explicarla sino que la introducen directamente desde la teoría de Brönsted, como muestra el siguiente ejemplo: 
62 "El proceso de hidrólisis lo introducimos como una reacción de transferencia 63 de protones entre un ion procedente de una sal y el agua. De esta forma 64 veremos el comportamiento ácido o básico de las disoluciones acuosas de las 65 sales neutras que no contiene H ni grupos $\mathrm{OH}^{\prime \prime}$ (Tomas).

3.2.2 Superposición de los niveles macroscópico y microscópico en la enseñanza de la neutralización

Para contrastar esta consecuencia utilizamos el ítem 5 del protocolo de análisis de libros de texto y el ítem 2 de la entrevista. Estas cuestiones tienen por objeto averiguar si en la enseñanza la definición macroscópica de los ácidos y las bases o de la neutralización se confunde con la interpretación conceptual de Arrhenius o de Brönsted. Para ello se analizaba en el texto o se le preguntaba al profesor el marco teórico en el que se introducían estos conceptos.

El $60 \%$ de los libros de texto no explicita el marco teórico en el que se introduce el concepto de neutralización.

2) ¿En qué marco teórico introduces la explicación de las reacciones de neutralización

3) Los estudiantes, frecuentemente indican que las reacciones ácido-base son reacciones de neutralización ¿Qué opinas sobre esta idea?

Los resultados de las preguntas 2 y 3 de la entrevista a los profesores sobre neutralización muestran que 11 de 20 dicen que explican este concepto según la teoría de Brönsted y el resto (9) según Arrhenius. No obstante, no parece que esté tan claro cuando se analizan sus explicaciones, como se muestra en el siguiente ejemplo:

75 "No sé si en realidad me baso en una teoría concreta, yo cuando les hablo de 76 reacciones de neutralización, le hablo de la reacción de disociación del 77 agua. Entonces a partir de la formación de agua, a partir de $\mathrm{H}$ o de $\mathrm{OH}$, es 78 cuando empiezo a hablar de reacción de neutralización. En general 79 consideramos neutralización al hecho de que sea alcanzado el punto neutro, 80 no la reacción en sí" (Ismael).

En las respuestas a la pregunta 3 nueve de los 20 profesores entrevistados consideran explícitamente que reacción ácido-base es igual a reacciones de neutralización como se muestra en el siguiente ejemplo:

77 “La verdad es que sí ya que el relacionar las reacciones ácido-base con la 78 neutralización es algo muy extendido. Yo mismo muchas veces, la verdad es 79 que lo uso indistintamente" (Beatriz).

La mitad de los entrevistados (10/20) no se definen o no contestan. Sólo 1 de 20 expresa que no se debe igualar reacción ácido-base a neutralización en el contexto de Brönsted. 
No es casualidad que el $60 \%$ de los libros de texto no indique el marco teórico en el que se introduce el concepto de neutralización y que en la entrevista sólo uno de 20 exprese que no se debe igualar reacción ácido-base a neutralización. Aunque es cierto que toda reacción de neutralización según la teoría de Arrhenius puede afirmarse que es una reacción ácido-base, en cambio según Brönsted la afirmación inversa no es cierta ya que cambian los conceptos de ácido, de base y de reacción ácido-base.

\subsection{3 ¿Se hace una introducción aproblemática de las teorías ácido-base en la enseñanza?}

Para contrastarla se proponen los ítems 5, 6 y 7 del protocolo de análisis de textos y la pregunta 4 de la entrevista a docentes. El objetivo de los mismos es averiguar si se considera necesario, al pasar de un perfil a otro en el estudio de los ácidos y las bases, presentar alguno de los problemas que no se pudieron resolver en la vieja teoría y, por tanto, se justifica la necesidad de introducir una nueva teoría que les dé solución.

De los resultados obtenidos en el protocolo aplicado a los textos se observa que un $37 \%$ de los libros no introducen la teoría de Arrhenius y el $63 \%$ de los libros que la presentan ninguno menciona, al menos, uno de los problemas que presentaba esta teoría (por ejemplo, la hidrólisis) y así hacer ver la necesidad de avanzar en la construcción de conocimientos, sustituyendo esta teoría por la de Brönsted -Lowry. En las respuestas a la pregunta 4 de la entrevista solamente hay 4 (de 20) profesores que desarrollan exclusivamente la teoría de

4) ¿Qué teorías ácido-base introduces en el estudio del tema?

¿Desarrollas la teoría de Arrhenius o pasas directamente a la de Brönsted? ¿Por qué?

Brönsted. Trece de 20 le dan la misma importancia a la teoría de Arrhenius que a la de Brönsted. Sólo la mitad de los profesores entrevistados indican razones que justifican el paso de una teoría a otra. En definitiva, esta visión aproblemática de las construcciones científicas puesta de manifiesto mediante el análisis de textos y entrevistas a docentes se transmite en la enseñanza.

En particular, la introducción aproblemática del concepto de hidrólisis se puede observar en los resultados obtenidos en el ítem 3 del protocolo y en la pregunta 1 de la entrevista (apartado 3.2.1). En ellos se observa que un $75 \%$ de los libros de texto y de los profesores entrevistados, no introducen la hidrólisis como proceso donde se presenta un comportamiento anómalo de las sales neutras al disolverse en agua en el marco de la teoría de Arrhenius, sino que directamente la definen en el perfil conceptual de la teoría de Brönsted.

\section{DISEÑOS Y RESULTADOS EXPERIMENTALES RELATIVOS AL APRENDIZAJE DE LAS REACCIONES ÁCIDO-BASE}

\subsection{Características generales del diseño relativo al aprendizaje de estas reacciones}

Para contrastar las dificultades que se pueden presentar en estos aprendizajes se han aplicado 3 cuestionarios con preguntas de respuesta abierta (sobre la predicción del 
carácter ácido-base de sustancias, sobre la neutralización y sobre la hidrólisis, respectivamente) a un total de 229 alumnos de Química de COU (18 años) de varios institutos de Bachillerato de Valencia durante 2 cursos escolares, desde 1996 a 1998. También se han realizado 2 entrevistas (sobre la neutralización y la hidrólisis) a 8 alumnos. Los instrumentos siempre se aplicaron después de impartir el tema de procesos ácido-base.

\subsection{Resultados encontrados en el aprendizaje}

\subsubsection{Los estudiantes no emplean la definición funcional o macroscópica para predecir el carácter ácido o básico de las disoluciones acuosas de sustancias}

Para contrastar esta dificultad se propone el cuestionario 1, cuyo objetivo es averiguar los criterios que usan los alumnos para predecir el carácter ácido-base de disoluciones acuosas de algunas sustancias. En la tabla 2 se presentan los porcentajes de acierto de cada uno de los ítems propuestos (Anexo 2).

La mayoría reconoce el carácter ácido del $\mathrm{H}_{2} \mathrm{SO}_{4}$ y el básico del $\mathrm{NaOH}(74 \%$ y66 \% de respuestas correctas, respectivamente), debido en parte a que ambas son sustancias bastante utilizadas en la enseñanza. A pesar de que el $\mathrm{NH}_{3}$ y el ácido acético son también sustancias frecuentes en un contexto escolar, en este caso el porcentaje de respuestas correctas baja a un poco más de la mitad (55\% y $54 \%$, respectivamente). Esto puede ser debido a que el amoníaco tiene $\mathrm{H}$ en su fórmula, el ácido acético $\mathrm{OH}$ en la suya y el alumno los disocia sin saber que no son disociables. El fracaso aumenta cuando las sustancias propuestas ya no son tan familiares y requieren cierto conocimiento de lo que son sustancias moleculares polares que se disuelven en agua pero que no se disocian, como es el caso del $\mathrm{CH}_{3} \mathrm{OH}$ (23\% de respuestas correctas) y el de la $\mathrm{CH}_{3} \mathrm{NH}_{2}(25 \%$ de respuestas correctas). Este fracaso aumenta mucho más en el caso del $\mathrm{SO}_{2}$, con sólo un $7 \%$ de respuestas correctas, esto se explica porque el $\mathrm{SO}_{2}$ no tiene ni $\mathrm{H}$ ni $\mathrm{OH}$ en su fórmula. Por otra parte, saber que su disolución acuosa es ácida requiere conocer que se trata del anhídrido del ácido sulfuroso.

En resumen, en las justificaciones dadas a sus respuestas se ha podido concluir que ningún estudiante utiliza la definición funcional de ácido o de base a partir de las propiedades de las sustancias. La mayoría de los estudiantes aplican un perfil conceptual próximo al de Arrhenius donde se generaliza la idea de ácido a toda sustancia que presenta en su fórmula átomos de $\mathrm{H}$ disociables o no y la de base, cuando hay grupos atómicos $\mathrm{OH}$.

\subsubsection{Para reconocerla neutralización los estudiantes memorizan el simbolismo ácido + base $\leftrightarrow$ sal + agua}

Para analizar si los estudiantes han comprendido el concepto de neutralización al menos en el perfil macroscópico y en uno de los teóricos que permiten su explicación, se ha elaborado el cuestionario 2 que consta de 2 ítems.

El ítem 1 del cuestionario 2 tiene un doble objetivo. En primer lugar, se pretende averiguar cuál es el concepto de neutralización que suele emplear el alumno. Esto es, a través de su respuesta se puede ver si reconoce el proceso como una neutralización mutua de las propiedades del ácido y la base (perfil macroscópico), una reacción entre los 
hidrogeniones del ácido y los iones oxhidrilos de la base (perfil de Arrhenius) o una transferencia protónica del ácido a la base. En este mismo ítem hay una segunda cuestión donde se pregunta cómo se puede comprobar experimentalmente que se trata de una neutralización. En cuanto al ítem 2 de este mismo cuestionario se ha elaborado para ver si el estudiantes es capaz de aplicar de manera significativa el perfil de Arrhenius para reconocer la neutralización entre 5 procesos seleccionados 0 , por el contrario, ha aplicado memorísticamente el simbolismo habitual del esquema ácido + base $\leftrightarrow$ sal + agua.

En la tabla 3 (Anexo 3) se presentan los resultados obtenidos al aplicar este cuestionario a 68 estudiantes. Respecto a la parte de tipo conceptual del ítem 1 se observa que un $70 \%$ de la muestra reconoce que es una neutralización. De ellos $44 \%$ utiliza el perfil macroscópico (ácido + base $\leftrightarrow$ sal + agua), $11 \%$ el de Arrhenius y el $15 \%$ no indica el marco teórico. $Y$ en cuanto a la parte de la pregunta de tipo procedimental solamente el $32 \%$ menciona el cambio de color de indicadores.

Referente al ítem 2, se intenta mostrar que la base de los errores está en que los alumnos han aprendido memorísticamente una representación simbólica del tipo ácido + base $\leftrightarrow$ sal + agua. Así, en la medida que las fórmulas de los dos reactivos de cada esquema de reacción se parezcan a un ácido y a una base, y en los productos aparezca una sal y agua, respectivamente, decidirán si es una reacción de neutralización. Se comprende que se equivoquen o duden mayoritariamente ( $2 / 3$ de la muestra) en los apartados $2 a$ y $2 b$ no clasificándolas como neutralización, debido al carácter absoluto de base que confieren al $\mathrm{Al}(\mathrm{OH})_{3}$ y al $\mathrm{Zn}(\mathrm{OH})_{2}$. No consideran la posibilidad de que estos compuestos puedan dar iones $\mathrm{H}^{+}$. Diferente sería el resultado si en estos esquemas hubiéramos utilizado las fórmulas $\mathrm{AlO}_{3} \mathrm{H}_{3}$ y $\mathrm{ZnO}_{2} \mathrm{H}_{2}$, respectivamente. También se explica que la mitad de las respuestas incorrectas en estos dos apartados considere que estos dos esquemas son hidrólisis. Ello seguramente es debido a que el simbolismo de reversibilidad (doble flecha) del esquema es considerado una igualdad y, entonces, los segundos miembros corresponden efectivamente a una hidrólisis. Hay sin embargo, una tercera parte de los equivocados que catalogan como disociaciones iónicas a estas reacciones porque las moléculas $\mathrm{Al}(\mathrm{OH})_{3}$ se parten dando $\mathrm{AlO}^{-}$. Por otra parte, y a pesar de que están en forma iónica los esquemas, se ve claramente que solo aproximadamente la tercera parte ( $37 \%$ en $2 a$ y $25 \%$ en 2 b) ha aplicado el perfil conceptual de Arrhenius al derivar correctamente que son neutralizaciones.

Coherentemente con lo dicho antes el análisis de los resultados de los apartados $2 \mathrm{~d}$ ( $9 \%$ de respuestas correctas) y $2 e$ ( $3 \%$ de respuestas correctas) muestra que se equivocan mayoritariamente al considerarlos como neutralizaciones debido a que se adaptan bastante bien al esquema ácido + base $\leftrightarrow$ sal + agua. Los estudiantes saben, casi todos, que el $\mathrm{HCl}$ es un ácido y un poco más de la mitad que el $\mathrm{NH}_{3}$ es una base. Por otra parte, creen coherentemente con los resultados obtenidos en el ítem 1 del cuestionario 1 que el metanol es una base, aunque también es posible que se disocie dando protones al tener de fórmula $\mathrm{CH}_{3} \mathrm{OH}$. El elevado porcentaje de errores obtenido en el apartado 2c) (sólo un 11\% contesta correctamente) también se explica por la ausencia de $\mathrm{H}$ y $\mathrm{OH}$ en las fórmulas de las sustancias reaccionantes y de $\mathrm{H}_{2} \mathrm{O}$ entre los productos.

En resumen, estos pobres resultados son el primer síntoma de confusión entre los procesos de neutralización, hidrólisis y disociación iónica. Y conforme vaya elevándose la dificultad cognitiva del proceso mayor será la confusión. 


\subsubsection{Los estudiantes mezclan los perfiles macroscópico y microscópico de referencia en los que se introducen los conceptos}

Para contrastar esta dificultad se propone la entrevista 1 con dos cuestiones que se preguntaron a 8 estudiantes de COU. El objetivo de la entrevista 1 es saber qué entiende el alumno por reacción de neutralización y cómo podría comprobar experimentalmente la neutralización entre el ácido clorhídrico $(\mathrm{HCl})$ y el hidróxido de sodio $(\mathrm{NaOH})$. También saber si consideraban que todas las reacciones ácido-base son de neutralización.

Entrevista $\left(\mathrm{N}^{0} 1\right)$ sobre el proceso de neutralización

1) ¿Qué es para ti una neutralización?

2)¿Es lo mismo para ti, reacción de neutralización que reacción ácido-base?

Explica tu respuesta.

En la pregunta 1, seis estudiantes emplean el perfil macroscópico, resultado que es convergente con el $70 \%$ obtenido en el ítem 1 del cuestionario 2 aplicado en 4.2 .2 Un estudiante aplica el perfil de Arrhenius y otro confunde neutralización con hidrólisis. En la pregunta 2, siete dicen que son idénticos los dos procesos y solamente uno dice que las reacciones de neutralización estarían incluidas en las de ácido-base de Brönsted.

\subsubsection{Los estudiantes confunden el proceso de hidrólisis de las sales con la neutralización o con una simple disociación iónica}

Para ver estas confusiones se ha elaborado un cuestionario ( 3 ) y la entrevista $2(\mathrm{~N}=8)$. El cuestionario 3 tiene dos ítems, el objetivo del primero es saber cómo definen los estudiantes la hidrólisis considerándose correctas tanto explicaciones macroscópicas como microscópicas. En cambio, el objetivo del ítem 2 de este cuestionario pretende averiguar si el alumno diferencia hidrólisis de neutralización o de la disociación iónica. La tabla 4 presenta los datos obtenidos en los ítems 1, 2a, 2b y 2c, respectivamente (Anexo 4).

Los resultados del ítem 1 muestran que sólo un $10 \%$ de la muestra de estudiantes encuestados $(\mathrm{N}=90)$ da respuestas correctas. La mitad de las respuestas incorrectas $(37 \%)$ indican que la hidrólisis es la ruptura de sustancias, compuestos, moléculas, etc. con el agua, pero no especifican que el compuesto que experimenta la hidrólisis es una sal.

También hay un elevado porcentaje de respuestas incorrectas (38\%) y en blanco (39\%) dadas al ítem 2a (disociación del ión amonio). Ello implica que el estudiante no asocia hidrólisis a reacción con el agua. Este resultado es coherente con el obtenido en el ítem anterior. La mitad de las respuestas incorrectas en el ítem $2 a$ señalan que es una hidrólisis porque interviene el agua sin dar más explicación. En el caso de la hidrólisis del carbonato (2b), la mitad de las respuestas incorrectas (37\%) se debe a que clasifican este proceso como neutralización argumentando que es un equilibrio y el símbolo de reversibilidad es el de igualdad. Es decir, el esquema ácido + base $\leftrightarrow$ sal + agua también se puede escribir como sal + agua $\leftrightarrow$ ácido + base, que es tal como está en $2 \mathrm{~b}$. En el caso de la disociación del $\mathrm{HCl}(2 \mathrm{c})$, casi todas las respuestas incorrectas (el $35 \%)$ indican 
que esta disociación iónica del $\mathrm{HCl}$ (según Arrhenius) para ellos es una hidrólisis, ya que es una reacción con el agua y el producto es ácido. Este resultado es coherente con el obtenido en el caso $2 a$.

En relación a la entrevista 2 elaborada, su objetivo concreto era saber si los estudiantes conocen qué reacción (ácida, básica o neutra) tendría una disolución acuosa de $\mathrm{Na}_{2} \mathrm{CO}_{3}$ y cómo lo comprobarían experimentalmente.

\footnotetext{
Entrevista ( $\left.\mathrm{N}^{\circ} 2\right)$ sobre la interpretación del proceso de hidrólisis

1) ¿Qué reacción (ácida, básica o neutra) tendría una disolución acuosa de $\mathrm{Na}_{2} \mathrm{CO}_{3}$ ?

2) ¿Cómo lo comprobarías experimentalmente?.

3) Tenemos un tubo de ensayo con $\mathrm{Na}_{2} \mathrm{CO}_{3}$ en disolución acuosa, vamos a agregar unas gotas de indicador, rojo de metilo. Además, tenemos papel indicador. ¿Qué crees que sucederá?

Se hace el experimento
}

En primer lugar, hay dos estudiantes que indican que la disolución del $\mathrm{Na}_{2} \mathrm{CO}_{3}$ en agua es neutra. Uno de ellos argumenta en el perfil macroscópico qué es una neutralización, como se muestra a continuación:

94 A. Pues supongo que sería una neutralización, porque es una sal y las

95 reacciones de neutralización de un ácido más una base dan una sal más

96 agua

97 E. ¿Cómo lo comprobarías experimentalmente?

97 A. Pues, juntando un ácido y una base para comprobarlo que me da. Creo 98 que sería ácido carbónico más sosa cáustica.

La otra alumna, Sonia, da una respuesta lógica derivada de la observación de la fórmula: es una sal neutra y por lo tanto su reacción también lo será.

Dos estudiantes más indican que la disolución es ácida pero no lo razonan. En tercer lugar, hay tres estudiantes que contestan que la disolución es básica. Dos de estos tres estudiantes argumentan lo siguiente:

113 A. A ver ... el ... ¿lo tengo que hacer de memoria? El Na ... tengo que

114 hacerlo por escrito ¿puedo? (la alumna escribe en el folio lo siguiente):

$115 \mathrm{~A} . \mathrm{Na}_{2} \mathrm{CO}_{3} \leftrightarrow 2 \mathrm{Na}^{+}+\mathrm{CO}_{3}{ }^{2-} ; \mathrm{y} \mathrm{Na}{ }^{+}+\mathrm{H}_{2} \mathrm{O} \leftrightarrow \mathrm{NaOH}+\mathrm{H}^{+}$;

$116 \mathrm{CO}_{3}^{2}+\mathrm{H}_{2} \mathrm{O} \leftrightarrow \mathrm{HCO}_{3}^{-}$; (tiene claro que el agua es un reactivo)

117 una sal neutra porque el Na seria un ion neutro porque proviene de una

118 ... sería el ácido conjugado de una base fuerte y el ion carbonato sería

119 ... procedería de un ácido débil ... entonces tendría carácter básico

120 yo creo que seria una sal básica. 
En resumen, de los ocho estudiantes entrevistados solamente hay 2 que claramente explicitan que una hidrólisis es la reacción entre los iones carbonato y el agua para dar iones hidroxilos y así explica la reacción básica de la disolución de la sal.

\section{CONCLUSIONES E IMPLICACIONES DIDÁCTICAS}

Los resultados obtenidos muestran que los profesores tenemos visiones distorsionadas sobre la naturaleza de la ciencia y sobre cómo aprenden los estudiantes. Además estas visiones se transmiten en la enseñanza agudizando las dificultades de aprendizaje. Así, las dificultades de los estudiantes para predecir el carácter ácido-base de las sustancias se explica porque cuando se enseña el tema no se enfatiza el nivel macroscópico de estos conceptos. En el mismo sentido se puede explicar que los estudiantes identifiquen neutralización según Arrhenius con reacción ácido-base de Brönsted. La visión aproblemática de las construcciones científicas explicaría que los estudiantes confundan hidrólisis con neutralización y disociación.

Las visiones distorsionadas de la ciencia pueden constituir un serio obstáculo en la renovación de la enseñanza, de ahí la importancia que se está dando a conocer y cuestionar el denominado pensamiento docente espontáneo (Gil 1991). Bell y Pearson (1992) señalan que no es posible cambiar lo que los profesores y alumnos hacen en la clase sin transformar su epistemología, sus concepciones acerca de cómo se construye el conocimiento y sus puntos de vista sobre la naturaleza de la ciencia.

Finalmente como profesores debemos plantearnos la necesidad de una enseñanza de estos conceptos científicos de manera que se facilite la superación de las dificultades de aprendizaje expuestas, se coadyuve a la construcción de los conceptos por los propios alumnos $\mathrm{y}$, al mismo tiempo, puedan adquirir una imagen más auténtica de la actividad científica. Una solución hipotética a este problema pasa por el diseño y desarrollo de estrategias de enseñanza/aprendizaje por investigación dirigida. Ésta será la meta de nuestro próximo trabajo.

\section{REFERENCIAS BIBLIOGRÁFICAS}

CROS, D., (1986). Conceptions of first-year university students of the constituents of matter and the notions of acids and bases, European Journal of Science Education, 8 (3), 305-313.

CROS, D., (1988). Conceptions of second year university students of some fundamental notions in chemistry, International Journal Science Education, 10 (3), 331.

DUIT, R., y PFUNDT, H., (1993). "Students Alternative Frameworks and Science Education", Institute for Science Education at the University of kiel, Germany.

FURIO,C. (1994). Tendencias actuales en la formación del profesorado de Ciencias. Enseñanza de las Ciencias, 12 (2), 188-199.

FURIO, C., AZCONA, R., GUISASOLA, J. y DOMÍNGUEZ, C. (1999). La enseñanzaaprendizaje del conocimiento químico. En F.J. Perales y P. Cañal (eds.), Didáctica de 
las Ciencias: Teoría y práctica de la Enseñanza de las Ciencias. Editorial Marfil: Alcoy (en prensa).

GABEL, D.L. (1998). The complexity of chemistry and implications for teaching. En B. Fraser and K. Tobin (Ed) International Handbook of Science Education. London: Kluwer.

GIL, D., (1991). ¿Qué han de saber y saber hacer los profesores de Ciencias? Enseñanza de las Ciencias, 9(1), 69-77.

GIL, D., (1993). Contribución de la Historia y Filosofía de las Ciencias a la transformación de la enseñanza de las Ciencias, Enseñanza de las Ciencias, 11(2), 197-202.

JOHNSTONE AH. (1993). The development of Chemistry Teaching. Journal of Chemical Education 70, 701-703.

KLEIN, U. (1994). Origin of the concept of chemical compound. Science in context, 7,16304.

KUHN, T.S., (1997). La Estructura de las Revoluciones Científicas, Breviarios, Fondo de Cultura Económica, España, 225.

MORTIMER, E, F., (1997). Para Além Das Fronteiras Da Química. RelaÇóes Entre Filosofía, Psicología e Ensino De Química. Quimica Nova, 20(2). 200-206.

NAKHLEH. M., (1994). Students' models of matter in the context of acid-base chemistry, Journal of Chemical Education, 71(6).

ROSS et al. (1991). Concept mapping and misconceptions: a study oh high school students' understanding of acids and bases. International Journal of Science Education, 13(1), 11-23.

SOLSONA, N. e IZQUIERDO, M. (1998). La conservación del elemento, una idea inexistente en el alumnado de Secundaria. Alambique, 17, 76-84.

SCHMIDT, H. J., (1991). A label as a hidder persuader: chemists neutralization concept, International Journal of Science Education, 13(4), 459-471.

VIDYAPATI, T. J. y SEETHARAMAPPA, J., (1995). Higher secondary school student's concepts of acids and bases, School Science Review, sept., 77 (278), 82, 84.

ZOLLER, U., (1990). Students misunderstanding and misconceptions in college freshman chemistry (General and Inorganic), Journal of Research in Science Teaching, 27(10), 1053-1065. 
Tabla 1. Porcentaje de libros de texto $(N=40)$ que cumplen cada una de las proposiciones consideradas en los ítems del protocolo.

\begin{tabular}{|c|c|}
\hline Contenido analizado & $\begin{array}{c}\text { Porcentaje } \\
(\%)\end{array}$ \\
\hline $\begin{array}{l}\text { 1) No se mencionan, al menos, } 3 \text { características principales de los ácidos y } \\
\text { de las bases que posteriormente se interpretarán con la teoría jónica (por } \\
\text { ejemplo, acción sobre indicadores, neutralización de propiedades y } \\
\text { conductividad eléctrica). } \\
\text { 2) Previamente a la introducción de cualquier explicación teórica: } \\
\text { No se proponen actividades procedimentales para clasificar en ácidas, } \\
\text { básicas o neutras algunas sustancias o productos. } \\
\text { 3) No se proponen ejemplos concretos de sustancias familiares que al } \\
\text { disolverse en agua presentan propiedades ácidas o básicas. } \\
\text { 4) No se presenta la hidrólisis de las sales neutras como proceso en el que } \\
\text { la disolución acuosa de estas sales tiene una reacción anómala (ácida o } \\
\text { básica). }\end{array}$ & $\begin{array}{l}82 \\
87\end{array}$ \\
\hline $\begin{array}{l}\text { 5) No se explícita el marco teórico en el que se introduce el concepto de } \\
\text { neutralización. } \\
\text { 6) No se introduce en el contenido del texto la teoría ácido-base de } \\
\text { Arrhenius. } \\
\text { 7) En caso de que se introduzcan las teorías de Arrhenius y de Brönsted, } \\
\text { no se menciona, al menos, un problema planteado a la teoría de Arrhenius } \\
\text { que haga ver la necesidad de avanzar en la construcción de conocimientos } \\
\text { hacia la teoría de Brönsted. }\end{array}$ & $\begin{array}{l}60 \\
37\end{array}$ \\
\hline $\begin{array}{l}\text { 8) No se propone al comienzo del tema, ningún ejemplo de relaciones CTS } \\
\text { próximas-al alumno que hagan ver el interés de su estudio. } \\
\text { 9) No se proponen a lo largo del tema, al menos, } 3 \text { ejemplos de relaciones } \\
\text { CTS próximas al alumno. }\end{array}$ & 63 \\
\hline
\end{tabular}

Tabla 2. Resultados porcentuales obtenidos $(N=86)$ al aplicar el cuestionario 1 sobre el carácter ácido o básico de soluciones acuosas de sustancias. 
Indica el tipo de reacción (ácida, básica o neutra) que presentará cada una de las siguientes sustancias en disolución acuosa y justifica tu respuesta en cada caso.

\begin{tabular}{|c|c|}
\hline Disolución acuosa de & $\begin{array}{c}\text { Porcentaje de respuestas } \\
\text { correctas (\%) }\end{array}$ \\
\hline 1. Amoníaco, $\mathrm{NH}_{3}$ & 55 \\
\hline 2. Metanol, $\mathrm{CH}_{3} \mathrm{OH}$ & 23 \\
\hline Sulfúrico, $\mathrm{H}_{2} \mathrm{SO}_{4}$ & 74 \\
\hline Sosa caústica, $\mathrm{NaOH}$ & 66 \\
\hline Acético, $\mathrm{CH}_{3} \mathrm{COOH}$ & 54 \\
\hline 6. Metilamina $\mathrm{CH}_{3} \mathrm{NH}_{2}$ & 25 \\
\hline 7. Dióxido de azufre $\mathrm{SO}_{2}(9)$ & 07 \\
\hline
\end{tabular}

Tabla 3. Porcentajes de respuestas correctas obtenidos en el cuestionario 2 sobre el proceso de neutralización $(N=68)$.

\begin{tabular}{|c|c|c|c|c|}
\hline Ítem & Referente a & $\begin{array}{l}(\%) \text { de } \\
\text { respuestas } \\
\text { correctas }\end{array}$ & $\begin{array}{l}\text { (\%) de } \\
\text { respuestas } \\
\text { incorrectas }\end{array}$ & $\begin{array}{c}(\%) \text { en } \\
\text { blanco }\end{array}$ \\
\hline 1 & $\begin{array}{l}\text { Se mezclan sendas disoluciones de un ácido } \\
\text { y un hidróxido. Explica si se produce alguna } \\
\text { reacción y cómo se puede comprobar } \\
\text { experimentalmente }\end{array}$ & $70 \%$ & $10 \%$ & $20 \%$ \\
\hline 2 & $\begin{array}{l}\text { Explica cuáles de los siguientes procesos se } \\
\text { pueden considerar de hidrólisis } \\
\text { neutralización o disociación: }\end{array}$ & & & \\
\hline $2 \mathrm{~A}$ & 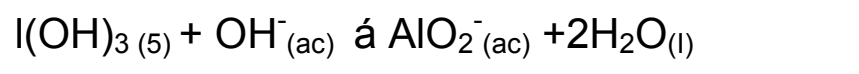 & $37 \%$ & $52 \%$ & $11 \%$ \\
\hline $2 b$ & $\mathrm{Zn}(\mathrm{OH})_{2(\mathrm{~s})}+2 \mathrm{OH}-(\mathrm{ac})$ á $\mathrm{ZnO}_{2}^{2-}{ }_{(\mathrm{ac})}+2 \mathrm{H}_{2} \mathrm{O}_{(\mathrm{l})}$ & $25 \%$ & $59 \%$ & $16 \%$ \\
\hline 2c & $\mathrm{SiO}_{2(\mathrm{~s})}+\mathrm{CaO}_{(\mathrm{s})}$ á $\mathrm{CaSiO}_{3}(\mathrm{~s})$ & $11 \%$ & $55 \%$ & $34 \%$ \\
\hline $2 d$ & $\mathrm{HCl}_{(\mathrm{g})}+\mathrm{CH}_{3} \mathrm{OH}_{(\mathrm{l})}$ á $\mathrm{CH}_{3} \mathrm{Cl}_{(\mathrm{g})}+\mathrm{H}_{2} \mathrm{O}_{(\mathrm{l})}$ & $9 \%$ & $58 \%$ & $33 \%$ \\
\hline $2 e$ & $\mathrm{NH}_{3(9)}+\mathrm{CH}_{3} \mathrm{OH}(\mathrm{l})$ á $\mathrm{CH}_{3} \mathrm{NH}_{2(\mathrm{~g})}+\mathrm{H}_{2} \mathrm{O}_{(\mathrm{l})}$ & $3 \%$ & $69 \%$ & $28 \%$ \\
\hline
\end{tabular}

Tabla 4. Porcentajes de respuesta correcta obtenidos en el cuestionario relativo al proceso de hidrólisis $(N=90)$.

\begin{tabular}{|l|l|c|c|c|}
\hline Ítem & Referente a & $\begin{array}{c}(\%) \text { de } \\
\text { respuestas } \\
\text { correctas }\end{array}$ & $\begin{array}{c}(\%) \text { de } \\
\text { respuestas } \\
\text { erróneas }\end{array}$ & $\begin{array}{c}(\%) \text { en } \\
\text { blanco }\end{array}$ \\
\hline
\end{tabular}




\begin{tabular}{|c|c|c|c|c|}
\hline 1) & $\begin{array}{l}\text { ¿Qué entiendes por hidrólisis?. Pon algún } \\
\text { ejemplo. }\end{array}$ & $10 \%$ & $74 \%$ & $14 \%$ \\
\hline 2) & $\begin{array}{l}\text { Explica cuáles de los siguientes procesos se } \\
\text { pueden considerar hidrólisis, neutralización o } \\
\text { disociación }\end{array}$ & & & \\
\hline 2a) & $\mathrm{NH}_{4}^{+}+\mathrm{H}_{2} \mathrm{O}$ á $\mathrm{NH}_{3(\mathrm{ac})}+\mathrm{H}_{3} \mathrm{O}^{+}{ }_{(\mathrm{ac})}$ & $23 \%$ & $38 \%$ & $39 \%$ \\
\hline $2 b)$ & $\mathrm{Na}_{2} \mathrm{CO}_{3(\mathrm{~s})}+2 \mathrm{HO}_{(\mathrm{l})}$ á $\mathrm{H}_{2} \mathrm{CO}_{3(\mathrm{ac})}+2 \mathrm{NaOH}_{(\mathrm{ac})}$ & $7 \%$ & $74 \%$ & $19 \%$ \\
\hline 2c) & $\mathrm{HCl}(\mathrm{ac})+\mathrm{H}_{2} \mathrm{O}_{(\mathrm{l})}$ á $\mathrm{Cl}^{-}{ }_{(\mathrm{ac})}+\mathrm{HO}_{(\mathrm{ac})}^{+}$ & $37 \%$ & $35 \%$ & $28 \%$ \\
\hline
\end{tabular}

